\title{
Mechanisms and Molecular Targets of the Tao-Hong-Si-Wu-Tang Formula for Treatment of Osteonecrosis of Femoral Head: A Network Pharmacology Study
}

\author{
Fanyu Fu $\mathbb{D}^{1},{ }^{1}$ Zeqing Huang, ${ }^{2}$ Hengli Ye $\mathbb{D}^{1},{ }^{1}$ Biao Tan $\mathbb{D},{ }^{2}$ Rongtian Wang $\mathbb{D},{ }^{3}$ \\ and Weiheng Chen $\mathbb{1}^{3}$ \\ ${ }^{1}$ Guizhou University of Traditional Chinese Medicine, No. 50, Shidonglu, Nanming District, Guizhou, Guiyang 550002, China \\ ${ }^{2}$ Department of Orthopaedics, Wangjing Hospital, China Academy of Chinese Medical Sciences, No. 6 Zhonghuannanlu, \\ Chaoyang District, Beijing 100102, China \\ ${ }^{3}$ Department of Orthopaedics, The Third Affiliated Hospital of Beijing University of Chinese Medicine, No. 51 Xiaoguan Street, \\ Chaoyang District, Beijing 100029, China
}

Correspondence should be addressed to Rongtian Wang; wrt135@163.com and Weiheng Chen; drchenweiheng@bucm.edu.cn

Received 13 March 2020; Revised 19 August 2020; Accepted 27 August 2020; Published 9 September 2020

Academic Editor: Adolfo Andrade-Cetto

Copyright $\odot 2020$ Fanyu Fu et al. This is an open access article distributed under the Creative Commons Attribution License, which permits unrestricted use, distribution, and reproduction in any medium, provided the original work is properly cited.

\begin{abstract}
The Tao-Hong-Si-Wu-Tang (THSWT) formula, a classic prescription of traditional Chinese medicine, has long been used for the treatment of osteonecrosis of femoral head (ONFH). However, its mechanisms of action and molecular targets are not comprehensively clear. In the present study, the Traditional Chinese Medicine System Pharmacology (TCMSP) database was employed to retrieve the active compounds of each herb included in the THSWT formula. After identifying the drug targets of active compounds and disease targets of ONFH, intersection analysis was conducted to screen out the shared targets. The protein-protein network of the shared targets was built for further topological analysis. Gene ontology and Kyoto Encyclopedia of Genes and Genomes pathway analysis were then carried out. A gene pathway network was constructed to screen the core target genes. We identified 61 active compounds, 155 drug targets, and 5443 disease targets. However, intersection analysis only screened out 37 shared targets. Kaempferol, luteolin, and baicalein regulated the greatest number of targets associated with ONFH. The THSWT formula may regulate osteocyte function through specific biological processes, including responses to toxic substances and oxidative stress. The regulated pathways included the relaxin, focal adhesion, nuclear factor- $\kappa \mathrm{B}$, toll-like receptor, and AGE/RAGE signaling pathways. RELA, VEGFA, and STAT1 were the important target genes in the gene network associated with the THSWT formula for the treatment of ONFH. Therefore, the present study suggested that the THSWT formula has an action mechanism involving multiple compounds and network targets for the treatment of ONFH.
\end{abstract}

\section{Introduction}

Osteonecrosis of femoral head (ONFH) represents a disruption of the blood supply to the femoral head due to trauma, corticosteroids, alcohol, and other ill-defined etiologies [1]. ONFH mainly affects individuals of working age [2]. It is estimated that there are more than 8 million patients with nontraumatic ONFH in China [3]. According to natural history studies, approximately half of all affected hips at pre- collapse stages (Association Research Circulation Osseous stage I or II) [4] would progress to irreversible collapse of the femoral head if left untreated [5]. Femoral head collapse can then progress to severe premature osteoarthritis of the hip, which is a common cause of lifelong disability and total hip arthroplasty in this active population [6].

To date, there are no optimal treatments for ONFH [7]. Total hip arthroplasty is not the first-choice treatment option since revision procedures and implant longevity remain 
tricky problems [8]. Other treatment modalities, commonly known as hip-preserving treatments, have demonstrated both favorable and poor outcomes $[9,10]$. According to a meta-analysis published in 2019, no marketed drugs are recommended for the treatment of ONFH [11].

During the past decade, Chinese experts have developed four versions of clinical guidelines, and traditional Chinese medicine (TCM) is consistently recommended as one of the main nonoperative treatments [12-15]. TCM holds a relatively unique point of view when treating ONFH, and blood stasis (Yu-Xue) is considered the pathological basis of ONFH [16]. According to the TCM theory, once the meridian branches (Jing-Luo) passing through the femoral head are blocked, the femoral head loses nutrition from qi and blood. Based on the blood stasis theory, the primary management strategy of TCM is to activate blood circulation (Huo-Xue-Fa) [15]. The Tao-Hong-Si-Wu-Tang (THSWT) formula is composed of Tao-Ren (Persicae Semen), Hong-Hua (Carthami Flos), Dang-Gui (Angelicae Sinensis Radix), ChuangXiong (Chuanxiong Rhizoma), Shu-Di-Huang (Rehmanniae Radix Praeparata), and Bai-Shao (Paeoniae Radix Alba), of which the main TCM function is to activate blood circulation. The THSWT formula is frequently administered in patients with ONFH in China. Data from animal testing suggest that the THSWT formula may help ameliorate the progression of steroid-induced avascular necrosis [17]. However, the active compounds and potential targets, as well as action pathways, remain poorly understood.

A general solution related to network pharmacology has been proposed recently, which has become a hot topic to investigate multiple molecular mechanisms of multipletarget compounds affecting biological networks for herbal medicines. Therefore, we employed network pharmacology to probe the pharmacological mechanisms of the THSWT formula against ONFH in this study.

\section{Materials and Methods}

2.1. Screening of Active Compounds. The THSWT formula consists of six Chinese herbs, including Tao-Ren (Persicae Semen), Hong-Hua (Carthami Flos), Dang-Gui (Angelicae Sinensis Radix), Chuang-Xiong (Chuanxiong Rhizoma), Shu-Di-Huang (Rehmanniae Radix Praeparata), and Bai-Shao (Paeoniae Radix Alba). The chemical compounds of these six herbs were identified using the Chinese Medicine System Pharmacology Database and Analysis Platform (TCMSP, http://tcmspw.com/tcmsp. php) [18]. TCMSP provides important data on the absorption-, distribution-, metabolism-, and excretion-related properties of Chinese herbs, including the oral bioavailability (OB), half-life, and drug-likeness (DL). In the present study, chemical compounds with OBs $\geq 30 \%$ and DLs $\geq 0.18$ were identified as active compounds. Eventually, 61 active compounds were screened out in total after removing duplications.

2.2. Identification of Drug Targets. The DrugBank (http:// www.drugbank.ca) [19] was employed to investigate potential targets of the 61 selected compounds. The DrugBank is a database containing approved drugs as well as experimental drugs. Finally, 587 drug targets were identified, including 102 in Persicae Semen, 257 in Carthami Flos, 55 in Angelicae Sinensis Radix, 30 in Rehmanniae Radix Praeparata, 104 in Paeoniae Radix Alba, and 39 in Chuanxiong Rhizoma. A total of 155 drug targets were collected after removing duplications. Protein sequences of these drug targets were normalized to official gene symbols using the UniProt database (https://www.uniprot.org/) [20].

2.3. Identification of Disease Targets. The differentially expressed genes associated with ONFH were downloaded from the GEO database (https://www.ncbi.nlm.nih.gov/geo/, Series: GSE74089, Samples: GSM1909502, GSM1909503, GSM1909504, GSM1909505, GSM1909506, GSM1909507, GSM1909508, GSM1909509) [21]. These original data were converted into a gene matrix using the Perl tool [22]. The collated data were analyzed using the Limma plugin of $\mathrm{R}$ software. Genes with a $P$-value $<0.05$ and $\mid \log _{2}$ (fold change)| $>1$ were identified as disease targets of ONFH.

\subsection{Protein-Protein Interaction Network Construction.} The Venny 2.1 online tool (http://bioinfogp.cnb.csic.es/ tools/venny/index.html) was used to draw a Venn diagram of drug targets and disease targets to obtain shared targets of the THSWT formula and ONFH. The shared target genes were then inputted into the String database (http:// string-db.org), with species limited to Homo sapiens and a confidence score $>0.4$, to construct the protein-protein interaction (PPI) network. The PPI network of drug targets and disease targets was visualized using Cytoscape software. Maximum Clique Centrality (MCC) is a network topology algorithm of the Cytohubba plugin, which helps identify core targets in the network. In the present study, the top 10 targets with the highest MCC scores were considered the core targets of the THSWT formula against ONFH.

2.5. Network Construction Method. An active compoundshared target network was constructed and visualized using Cytoscape 3.7.2 software. The core compounds and core targets in this network were automatically identified. Each node in the network represented an active compound or shared target. The edge between two nodes implied that a particular compound might act on the target connected with it. The topological parameters of each node, including the degree, betweenness, and closeness, were calculated and used as screening criteria for the crucial nodes. Overall, nodes with greater parameter values were recognized as crucial nodes of the THSWT formula against ONFH. In the present study, a key compound was required to fulfill the criterion that these three parameters exceeded the median of the selected compounds.

2.6. Bioinformatics Analysis. Gene ontology (GO) biological process (BP) enrichment analysis and Kyoto Encyclopedia of Genes (KEGG) pathway enrichment analysis were 
conducted using the David 6.8 database (https://david. ncifcrf.gov/). During these procedures, P.adjust $<0.05$ suggested statistical significance in the enrichment degree. The top $20 \mathrm{GO}$ and top 20 KEGG results with the lowest P.adjust values were displayed in the form of bubble charts using R-studio software. The genes with significantly regulated pathways were selected for gene pathway network analysis to screen out the key target genes of the THSWT formula in the treatment of ONFH.

\section{Results}

3.1. Active Compounds and Shared Targets. Sixty-one chemical compounds of the THSWT formula (Table 1) were identified as the active compounds. The distribution of differentially expressed genes was displayed using volcanic maps (Figure 1). Data of upregulated genes were shown as red dots, and downregulated genes were shown as green dots. A total of 5443 differentially expressed genes in ONFH were collected from the GEO database, including 3291 upregulated genes and 2152 downregulated genes. Intersection analysis of 155 drug target genes and 5443 disease target genes identified 37 shared targets (Figure 2). These 37 targets were considered potential targets of the THSWT formula for the treatment of ONFH.

3.2. PPI Network Analysis. The PPI network (Figure 3) contained 37 nodes, which corresponded to 37 shared targets, and 120 edges that represented the target-target interactions. The top 10 target genes with the highest MCC scores were VEGFA, PTGS2, CCND1, JUN, RELA, STAT1, $A H R, N R 3 C 1, M C L 1$, and MMP2, which were considered the core targets (Figure 4).

3.3. Compound-Shared Target Network Analysis. The compound-shared target network (Figure 5) contained 67 nodes, which corresponded to 30 candidate compounds, 37 shared targets, and 94 edges representing the compound-target interactions (Table 2). Topological calculations revealed nine compounds fulfilling the criteria with all parameter values (degree, betweenness, and closeness) exceeding the median of the 30 selected compounds (Table 3). Overall, kaempferol, luteolin, and baicalein were found to act on the top three greatest numbers of targets $(15,14$, and 8 targets, respectively). In addition, the OBs of kaempferol, luteolin, and baicalein were $41.88 \%, 36.16 \%$, and $33.52 \%$, respectively. Therefore, they were considered the key compounds in the THSWT formula for the treatment of ONFH.

3.4. GO and KEGG Pathway Enrichment Analyses. BP, cellular component (CC), and molecular function (MF) analyses of the 37 target genes revealed 603 GO terms that were significantly enriched, including 540 in BP, 23 in CC, and 40 in MF analyses. The GO terms with the top 20 lowest P.adjust values are shown in Figure 6.

KEGG pathway analysis revealed 78 pathways that were significantly enriched. The top 20 terms are shown in
Figure 7. The clinically significant pathways in the top 20 included the relaxin, focal adhesion, nuclear factor $(\mathrm{NF})-\kappa \mathrm{B}$, Toll-like receptor (TLR), and AGE/RAGE signaling pathways.

Finally, the gene pathway network was constructed based on the significantly enriched pathways and genes that regulated these pathways, as presented in Figure 8. Topological analysis of 20 pathways and 21 genes was carried out. The squares represented target genes, and the V-shapes represented pathways in the network. The network diagram suggested that RELA had the maximum degree (number of connected nodes) and thus was considered the core target. Several other targets also had more significant degrees, such as JUN, VEGFA, and CCND1.

\section{Discussion}

TCM holds a similar view that ischemia of the femoral head is a key pathological change in ONFH. Chinese herbal medications with the TCM function of activating blood (Huo-Xue-Fa) have been consistently recommended by Chinese guidelines as an important nonoperative treatment for ONFH [12-15]. The THSWT formula, as a basic prescription to implement the therapeutic principle of activating blood [23], has demonstrated promising effects in ameliorating the progression of ONFH [24]. However, the biological activity of the THSWT formula remains poorly understood, particularly regarding whether it can increase the blood supply to the femoral head and whether it possesses any bone protective activity. Data from the present study suggest that the THSWT formula contains multiple active compounds that act on a network of different targets by regulating a number of signaling pathways, which contribute to the implementation of the THSWT formula in clinical practice.

An updated meta-analysis concluded that marketed drugs fail to prevent the progression of ONFH [11], but an increasing number of clinical studies on TCM have demonstrated promising outcomes $[25,26]$. Essentially, TCM prescribes several natural compounds, most of which are still not approved as marketed productions. However, this can be an important way to discover potential drugs for ONFH. In the present study, kaempferol, luteolin, and baicalein were among the important active compounds of the THSWT formula, since these compounds can act on 15, 14, and 8 different disease targets, respectively. Kaempferol is a common flavonol present in Chinese herbs with therapeutic properties, including antioxidant and anti-inflammatory activities [27]. Recent studies have suggested that kaempferol also has bone protective activity, since animal testing has found that kaempferol antagonizes the apoptotic effect of dexamethasone on osteoblasts [28]. Both isolated luteolin and extracts from luteolin-rich plants exhibit anti-inflammatory activity [29]. Luteolin also helps inhibit the bone resorption induced by mature osteoclasts [30]. A number of studies have demonstrated that baicalein has potent neuroprotective properties [31]. Additionally, baicalein inhibits the bone resorptive activity of mature osteoclasts by inducing apoptosis [32]. We can easily conclude that the 
TABLE 1: The list of active compounds in the THSWT formula.

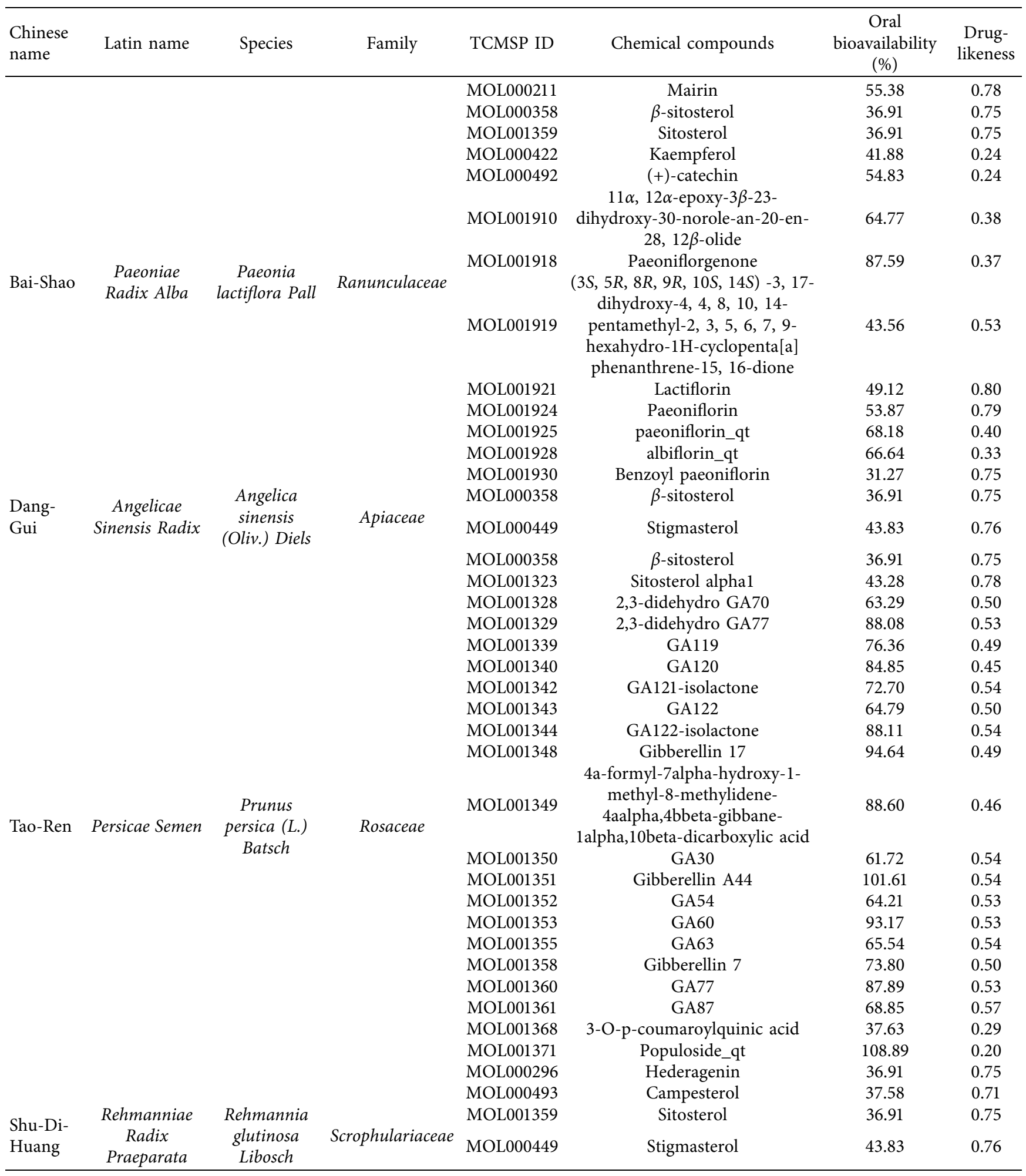


TABle 1: Continued.

\begin{tabular}{|c|c|c|c|c|c|c|c|}
\hline $\begin{array}{l}\text { Chinese } \\
\text { name }\end{array}$ & Latin name & Species & Family & TCMSP ID & Chemical compounds & $\begin{array}{c}\text { Oral } \\
\text { bioavailability } \\
(\%) \\
\end{array}$ & $\begin{array}{l}\text { Drug- } \\
\text { likeness }\end{array}$ \\
\hline \multirow{7}{*}{$\begin{array}{l}\text { Chuang- } \\
\text { Xiong }\end{array}$} & \multirow{7}{*}{$\begin{array}{c}\text { Chuanxiong } \\
\text { Rhizoma }\end{array}$} & \multirow{7}{*}{$\begin{array}{l}\text { Ligusticum } \\
\text { chuanxiong } \\
\text { Hort }\end{array}$} & \multirow{7}{*}{ Apiaceae } & MOL001359 & Sitosterol & 36.91 & 0.75 \\
\hline & & & & MOL000433 & Folic acid & 68.96 & 0.71 \\
\hline & & & & MOL001494 & Mandenol & 42.00 & 0.19 \\
\hline & & & & MOL002135 & Myricanone & 40.60 & 0.51 \\
\hline & & & & MOL002140 & Perlolyrine & 65.95 & 0.27 \\
\hline & & & & MOL002151 & Senkyunone & 47.66 & 0.24 \\
\hline & & & & MOL002157 & Wallichilide & 42.31 & 0.71 \\
\hline \multirow{22}{*}{$\begin{array}{l}\text { Hong- } \\
\text { Hua }\end{array}$} & \multirow{22}{*}{ Carthami Flos } & \multirow{22}{*}{$\begin{array}{l}\text { Carthamus } \\
\text { tinctorius L. }\end{array}$} & \multirow{22}{*}{ Compositae } & MOL000358 & $\beta$-sitosterol & 36.91 & 0.75 \\
\hline & & & & MOL000449 & Stigmasterol & 43.83 & 0.76 \\
\hline & & & & MOL000422 & Kaempferol & 41.88 & 0.24 \\
\hline & & & & MOL001771 & $\begin{array}{c}\text { Poriferast-5-en-3beta-ol } \\
\text { 4-[(E)-4-(3,5-dimethoxy-4-oxo- } \\
\text { 1-cyclohexa-2,5-dienylidene) }\end{array}$ & 36.91 & 0.75 \\
\hline & & & & MOL002694 & $\begin{array}{c}\text { but-2-enylidene]-2,6- } \\
\text { dimethoxycyclohexa-2,5-dien-1- } \\
\text { one }\end{array}$ & 48.47 & 0.36 \\
\hline & & & & MOL002680 & Flavoxanthin & 60.41 & 0.56 \\
\hline & & & & MOL002695 & Lignan & 43.32 & 0.65 \\
\hline & & & & MOL002698 & Lupeol-palmitate & 33.98 & 0.32 \\
\hline & & & & MOL002706 & Phytoene & 39.56 & 0.50 \\
\hline & & & & MOL002707 & Phytofluene & 43.18 & 0.50 \\
\hline & & & & MOL002710 & Pyrethrin II & 48.36 & 0.35 \\
\hline & & & & MOL002712 & 6-Hydroxykaempferol & 62.13 & 0.27 \\
\hline & & & & MOL002714 & Baicalein & 33.52 & 0.21 \\
\hline & & & & MOL002717 & qt_carthamone & 51.03 & 0.20 \\
\hline & & & & MOL002719 & 6-Hydroxynaringenin & 33.23 & 0.24 \\
\hline & & & & MOL002721 & Quercetagetin & 45.01 & 0.31 \\
\hline & & & & MOL002757 & $\begin{array}{l}\text { 7,8-dimethyl-1H-pyrimido[5,6- } \\
\text { g]quinoxaline-2,4-dione }\end{array}$ & 45.75 & 0.19 \\
\hline & & & & MOL002773 & Beta-carotene & 37.18 & 0.58 \\
\hline & & & & MOL002776 & Baicalin & 40.12 & 0.75 \\
\hline & & & & MOL000006 & Luteolin & 36.16 & 0.25 \\
\hline & & & & MOL000953 & CLR & 37.87 & 0.68 \\
\hline & & & & MOL000098 & Quercetin & 46.43 & 0.28 \\
\hline
\end{tabular}

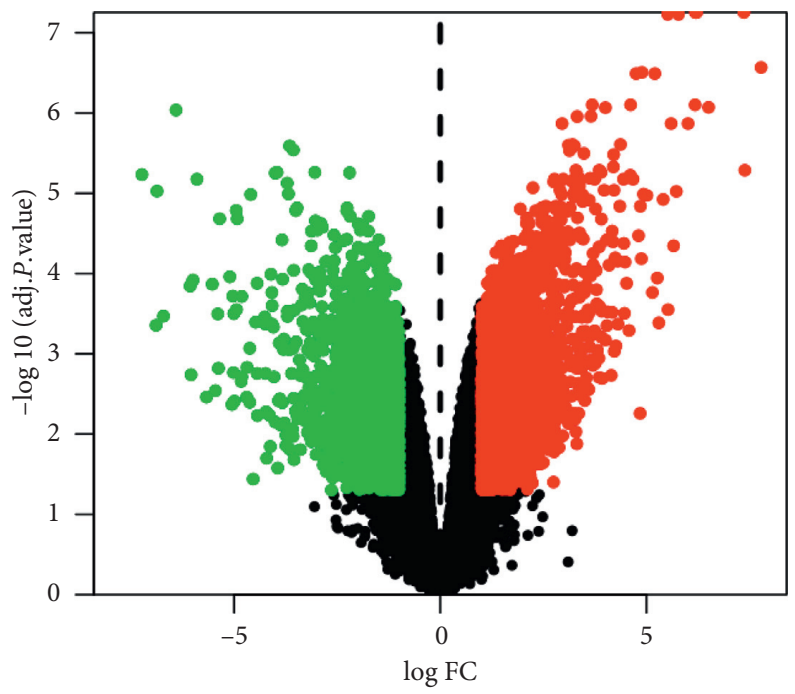

FIGURE 1: Volcano map of differently expressed genes. Green dots represent the downregulated genes, and red dots represent the upregulated genes. 


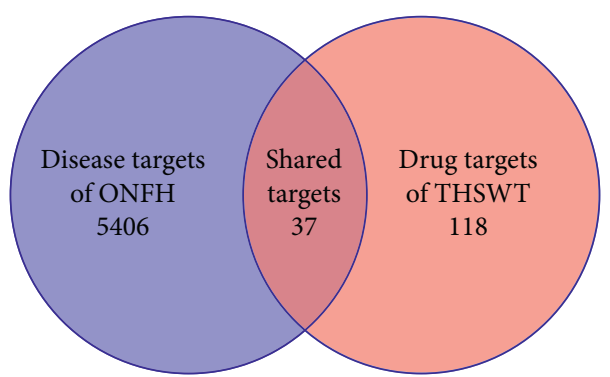

FIgURE 2: Venn diagram showing the intersection of drug targets of the THSWT formula and disease targets of osteonecrosis of femoral head.

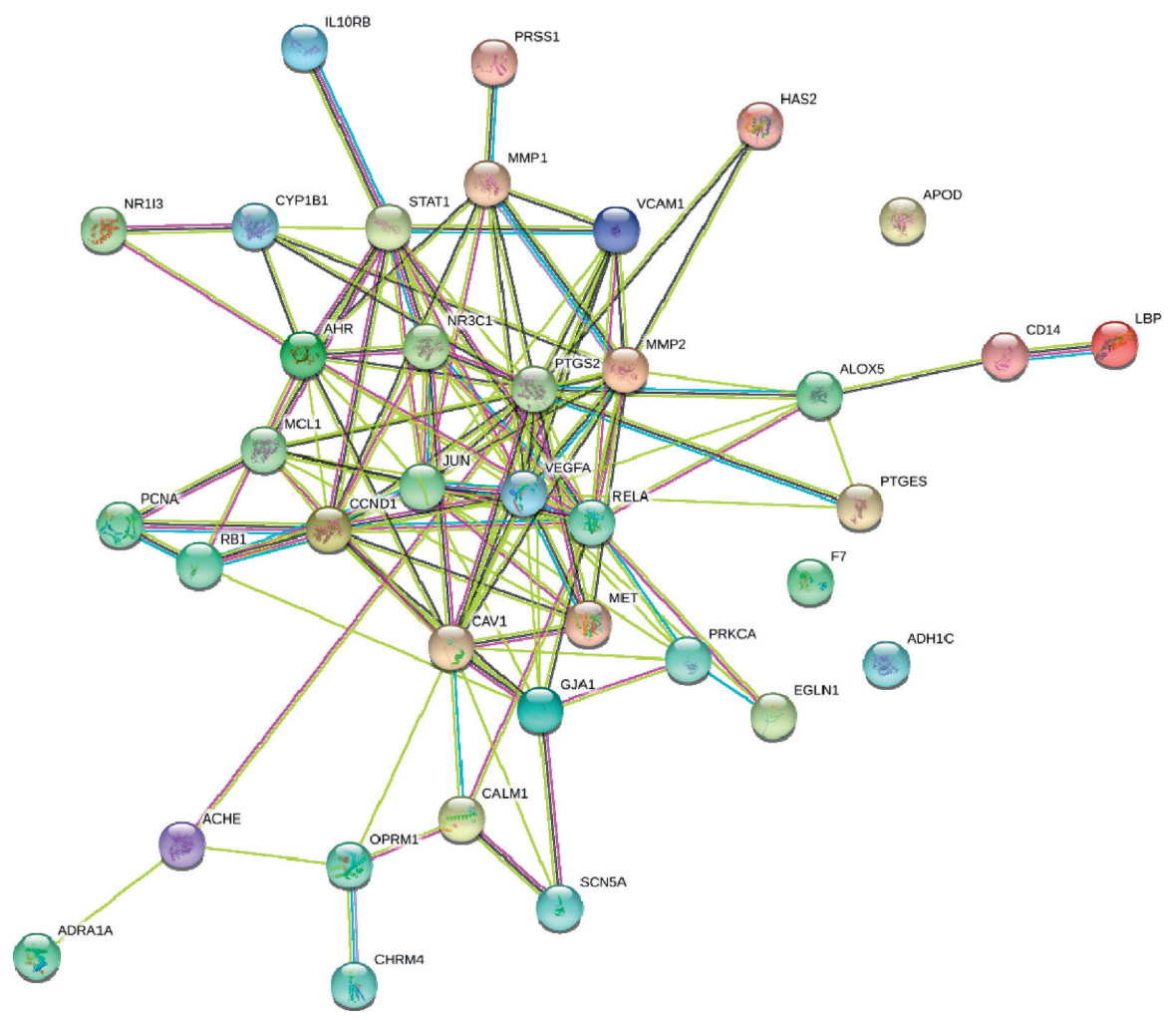

FIGURE 3: Protein-protein interaction network of the 37 shared targets.

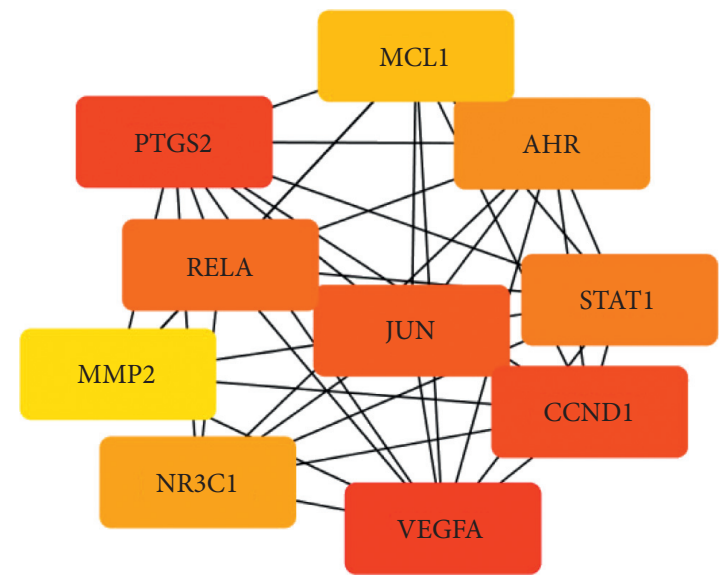

FIgURE 4: Protein-protein interaction network of the top 10 shared targets with highest MCC scores. The shade of color is proportional to the topological importance, with red targets indicating more important positions. 


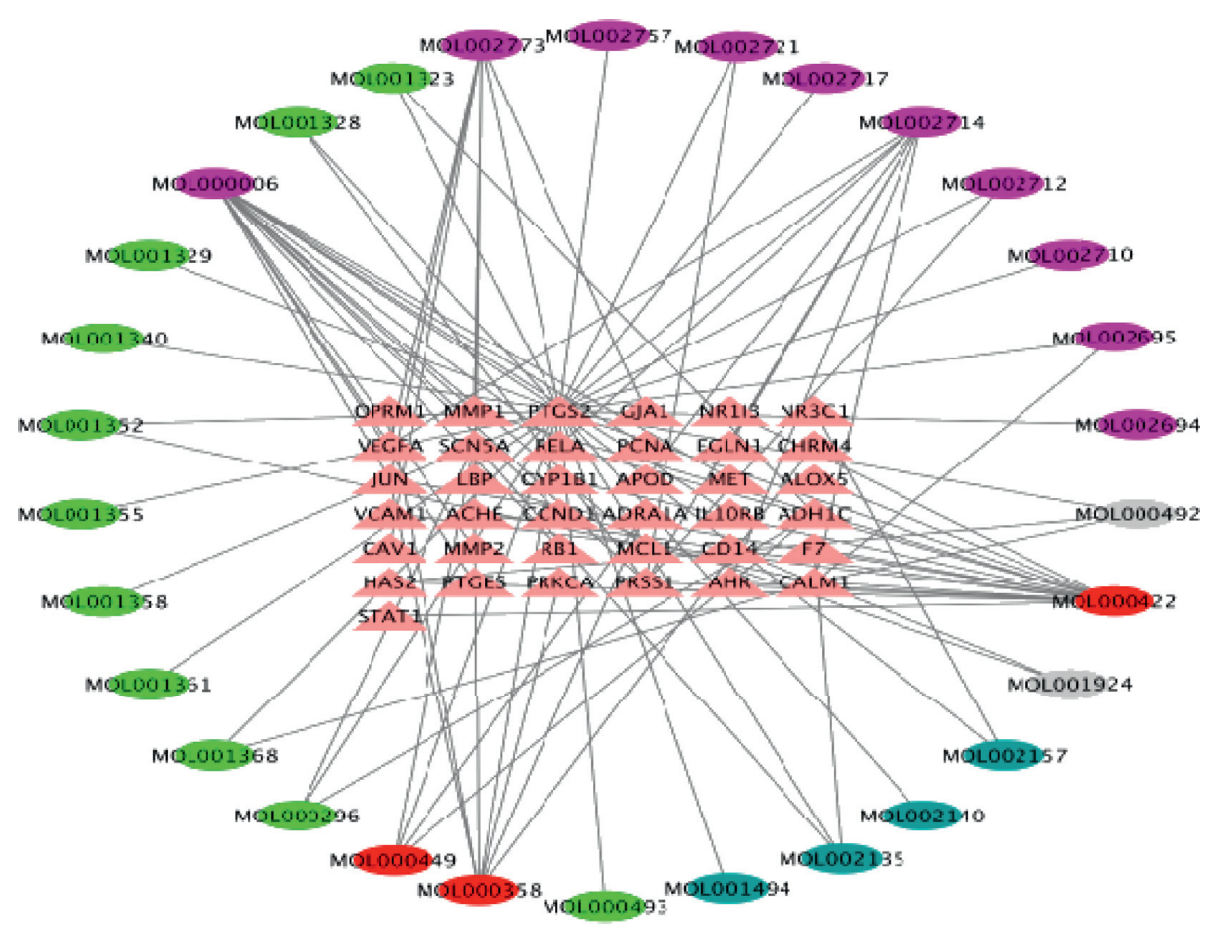

FIGURE 5: The compound-shared targets network. The ovals represent the chemical compounds, the colors represent the herb sources of chemical compounds, and the pink triangles represent the 37 shared genes. The gray ovals represent Paeoniae Radix Alba, the dark green ovals represent Chuanxiong Rhizoma, the purple ovals represent Carthami Flos, the light green ovals represent Persicae Semen, and the red ovals represent chemical compounds with multiple herb sources.

natural compounds of the THSWT formula, particularly the three aforementioned compounds, confer bone protective activity and have high $\mathrm{OB}$ scores; they are likely to be the core compounds for the treatment of ONFH.

GO enrichment analysis suggested that the THSWT formula regulates a variety of BPs and affects various CCs and MFs. Cellular responses to toxic substances and oxidative stress are important BPs involved in the development of ONFH. Corticosteroids and alcohol are key toxic substances that cause ONFH. Previous studies have confirmed that the rs1045642 single-nucleotide polymorphism of $A B C B 1$, an important determinant in the elimination of toxic substances, is closely associated with the occurrence of steroid-induced ONFH [33]. Moreover, oxidative stress plays a role in the activation of coagulation, which is the underlying BP that leads to ischemia of the femoral head [34]. Our data showed that membrane raft and membrane microdomains are among the most significant CCs affected by the THSWT formula. Additionally, the significantly mediated MFs include protein heterodimerization activity and proximal promoter sequence-specific DNA binding.

KEGG enrichment analysis suggested that the THSWT formula may regulate various signaling pathways. The relaxin, focal adhesion, and $\mathrm{NF}-\kappa \mathrm{B}$ signaling pathways are enriched pathways with important clinical significance. The relaxin signaling pathway is a potent stimulator of osteoclastogenesis from hematopoietic precursors, which regulate the activity of mature osteoclasts [35]. Focal adhesion kinase (FAK) is a nonreceptor protein tyrosine kinase and scaffolding protein that mediates numerous cellular functions, including adhesion, migration, and invasion. FAK inhibitors reduce synovial fibroblast invasion and migration [36]; thus, inhibition of FAK may help ameliorate the bone marrow edema and synovitis observed in the development of ONFH. TLR antagonists can be used for the treatment of inflammatory and autoimmune diseases, which also inhibit the activation of NF- $\kappa \mathrm{B}$. NF- $\kappa \mathrm{B}$, one of the most important transcriptional signaling molecules, participates in downstream inflammatory pathways and the TLR signaling pathway. The essential role of NF- $\kappa \mathrm{B}$ in osteoclastogenesis has been demonstrated genetically. NF- $\kappa \mathrm{B}$ can transduce signals by recruiting adaptor molecules. In addition, NF- $\kappa \mathrm{B}$ can induce the proliferation of monocytes/macrophages, which finally form osteoclasts [37]. Our data also suggested that the biological activity of the THSWT formula is associated with a number of pathways involved in other diseases, including infections, cancers, and diabetes-related complications. Interestingly, the AGE/RAGE signaling pathway is the most enriched pathway based on our data. AGE/RAGE signaling is a well-studied cascade in many different disease states; inhibition of the AGE/RAGE system may be a promising target for therapeutic intervention for vascular complications such as acquired blindness, endstage renal failure, a variety of neuropathies, and 
TABLE 2: The list of targets and active compounds of Tao-Hong-Si-Wu-Tang formula against ONFH.

\begin{tabular}{|c|c|c|c|}
\hline TCMSP ID & Chemical compound & Herb source & Target \\
\hline MOL000006 & Luteolin & Carthami Flos & $\begin{array}{c}\text { PTGS2, PRSS1, RELA, VEGFA, } \\
\text { CCND1, MMP2, RB1, JUN, IL6, MMP1, } \\
\text { PCNA, MCL1, PTGES, MET }\end{array}$ \\
\hline MOL000296 & Hederagenin & Persicae Semen & ADH1C, SCN5A, PTGS2 \\
\hline MOL000358 & Gibberellin 7 & $\begin{array}{c}\text { Paeoniae Radix Alba, Angelicae } \\
\text { Sinensis Radix, Carthami Flos, } \\
\text { Persicae Semen }\end{array}$ & $\begin{array}{l}\text { PTGS2, SCN5A, CHRM4, ADRA1A, } \\
\text { OPRM1, JUN, PRKCA }\end{array}$ \\
\hline MOL000422 & Kaempferol & $\begin{array}{c}\text { Paeoniae Radix Alba, Carthami } \\
\text { Flos }\end{array}$ & $\begin{array}{l}\text { PTGS2, PRSS1, ACHE, F7, CALM1, } \\
\text { RELA, JUN, MMP1, STAT1, VCAM1, } \\
\text { CYP1B1, ALOX5, HAS2, AHR, NR1I3 }\end{array}$ \\
\hline MOL000449 & Stigmasterol & $\begin{array}{c}\text { Rehmanniae Radix Praeparata, } \\
\text { Angelicae Sinensis Radix, } \\
\text { Carthami Flos }\end{array}$ & ADH1C, PTGS2, SCN5A, ADRA1A \\
\hline MOL000492 & $(+)$-catechin & Paeoniae Radix Alba & PTGS2, CALM1, HAS2 \\
\hline MOL000493 & Campesterol & Persicae Semen & PTGS2 \\
\hline MOL001323 & Sitosterol alpha1 & Persicae Semen & PTGS2, ADH1C \\
\hline MOL001328 & 2,3-didehydro GA70 & Persicae Semen & PTGS2, PRSS1 \\
\hline MOL001329 & 2,3-didehydro GA77 & Persicae Semen & PTGS2 \\
\hline MOL001340 & GA120 & Persicae Semen & PTGS2 \\
\hline MOL001352 & GA54 & Persicae Semen & PTGS2, CALM1 \\
\hline MOL001355 & GA63 & Persicae Semen & PTGS2 \\
\hline MOL001358 & Gibberellin 7 & Persicae Semen & PTGS2 \\
\hline MOL001361 & GA87 & Persicae Semen & PTGS2 \\
\hline MOL001368 & 3-O-p-coumaroylquinic acid & Persicae Semen & PTGS2, CALM1 \\
\hline MOL001494 & Mandenol & Chuanxiong Rhizoma & PTGS2 \\
\hline MOL001924 & Paeoniflorin & Paeoniae Radix Alba & IL6, CD14, LBP \\
\hline MOL002135 & Myricanone & Chuanxiong Rhizoma & SCN5A, PTGS2, F7 \\
\hline MOL002140 & Perlolyrine & Chuanxiong Rhizoma & PTGS2 \\
\hline MOL002157 & Wallichilide & Chuanxiong Rhizoma & PTGS2, NR3C1 \\
\hline MOL002694 & $\begin{array}{l}\text { 4-[(E)-4-(3,5-dimethoxy-4-oxo-1-cyclohexa- } \\
\text { 2,5-dienylidene) but-2-enylidene]-2,6- } \\
\text { dimethoxycyclohexa-2,5-dien-1-one }\end{array}$ & Carthami Flos & PTGS2 \\
\hline MOL002695 & Lignan & Carthami Flos & PTGS2, CALM1 \\
\hline MOL002710 & Pyrethrin II & Carthami Flos & PTGS2 \\
\hline MOL002712 & 6-hydroxykaempferol & Carthami Flos & PTGS2, PRSS1 \\
\hline MOL002714 & Baicalein & Carthami Flos & $\begin{array}{l}\text { PTGS2, PRSS1, CALM1, RELA, } \\
\text { VEGFA, AHR, EGLN1, APOD }\end{array}$ \\
\hline MOL002717 & qt_carthamone & Carthami Flos & PTGS2 \\
\hline MOL002721 & Quercetagetin & Carthami Flos & PTGS2, PRSS1 \\
\hline MOL002757 & $\begin{array}{l}\text { 7,8-dimethyl-1H-pyrimido[5,6-g]quinoxaline- } \\
\text { 2,4-dione }\end{array}$ & Carthami Flos & PTGS2 \\
\hline MOL002773 & Beta-carotene & Carthami Flos & $\begin{array}{c}\text { VEGFA, MMP2, JUN, PTGS2, MMP1, } \\
\text { CAV1, GJA1 }\end{array}$ \\
\hline
\end{tabular}

accelerated atherosclerosis [38]. The AGE/RAGE signaling pathway also plays an important physiological role in the regulation of skeletal development, homeostasis, and repair/regeneration [39].

Gene pathway network analysis revealed that RELA, $V E G F A$, and STAT1 were among the core targets of the THSWT formula in the treatment of ONFH. RELA is a member of the NF- $\kappa \mathrm{B} / \mathrm{Rel}$ family. The transcription factor $\mathrm{NF}-\kappa \mathrm{B}$ is a critical regulator of immune and inflammatory responses. Mice lacking RelA/p65 in the hematopoietic compartment have been shown to have a deficient osteoclastogenic response to RANKL and are protected from arthritis-induced osteolysis. It has been shown that inhibition of NF- $\kappa \mathrm{B}$ is an effective approach to inhibit osteoclast formation and bone resorptive activity [40]. The vascular endothelial growth factor A (VEGFA) gene is located on chromosome $6 \mathrm{p} 31.3$ [41]. It encodes a member of vascular endothelial growth factor. Several previous studies have linked multiple genetic polymorphisms within the promoter region of VEGFA to the disease status of 
TABLE 3: Topological parameters of candidate compounds for THSWT against ONFH.

\begin{tabular}{|c|c|c|c|c|}
\hline TCMSP ID & Chemical compound & Degree & Betweenness & Closeness \\
\hline MOL000422 & Kaempferol & 15 & 1080.90 & 36.32 \\
\hline MOL000006 & Luteolin & 14 & 1370.81 & 36.17 \\
\hline MOL002714 & Baicalein & 8 & 394.46 & 31.65 \\
\hline MOL000358 & Gibberellin 7 & 7 & 503.66 & 30.98 \\
\hline MOL002773 & Beta-carotene & 7 & 365.76 & 30.98 \\
\hline MOL000449 & Stigmasterol & 4 & 120.36 & 28.98 \\
\hline MOL000296 & Hederagenin & 3 & 66.29 & 28.32 \\
\hline MOL002135 & Myricanone & 3 & 65.72 & 28.32 \\
\hline MOL000492 & $(+)$-catechin & 3 & 49.35 & 28.32 \\
\hline MOL001924 & Paeoniflorin & 3 & 258.00 & 19.03 \\
\hline MOL002157 & Wallichilide & 2 & 130.00 & 27.65 \\
\hline MOL001323 & Sitosterol alpha1 & 2 & 38.77 & 27.65 \\
\hline MOL001352 & GA54 & 2 & 11.67 & 27.65 \\
\hline MOL001368 & 3-O-p-coumaroylquinic acid & 2 & 11.67 & 27.65 \\
\hline MOL002695 & Lignan & 2 & 11.67 & 27.65 \\
\hline MOL001328 & 2,3-didehydro GA70 & 2 & 9.63 & 27.65 \\
\hline MOL002712 & 6-hydroxykaempferol & 2 & 9.63 & 27.65 \\
\hline MOL002721 & Quercetagetin & 2 & 9.63 & 27.65 \\
\hline MOL001329 & 2,3-didehydro GA77 & 1 & 0.00 & 26.98 \\
\hline MOL001340 & GA120 & 1 & 0.00 & 26.98 \\
\hline MOL001355 & GA63 & 1 & 0.00 & 26.98 \\
\hline MOL001358 & Gibberellin 7 & 1 & 0.00 & 26.98 \\
\hline MOL001361 & GA87 & 1 & 0.00 & 26.98 \\
\hline MOL000493 & Campesterol & 1 & 0.00 & 26.98 \\
\hline MOL001494 & Mandenol & 1 & 0.00 & 26.98 \\
\hline MOL002140 & Perlolyrine & 1 & 0.00 & 26.98 \\
\hline MOL002694 & $\begin{array}{l}\text { 4-[(E)-4-(3,5-dimethoxy-4-oxo-1-cyclohexa-2,5-dienylidene) but-2-enylidene]-2,6- } \\
\text { dimethoxycyclohexa-2,5-dien-1-one }\end{array}$ & 1 & 0.00 & 26.98 \\
\hline MOL002710 & Pyrethrin II & 1 & 0.00 & 26.98 \\
\hline MOL002717 & qt_carthamone & 1 & 0.00 & 26.98 \\
\hline MOL002757 & 7,8-dimethyl-1H-pyrimido[5,6-g]quinoxaline-2,4-dione & 1 & 0.00 & 26.98 \\
\hline
\end{tabular}

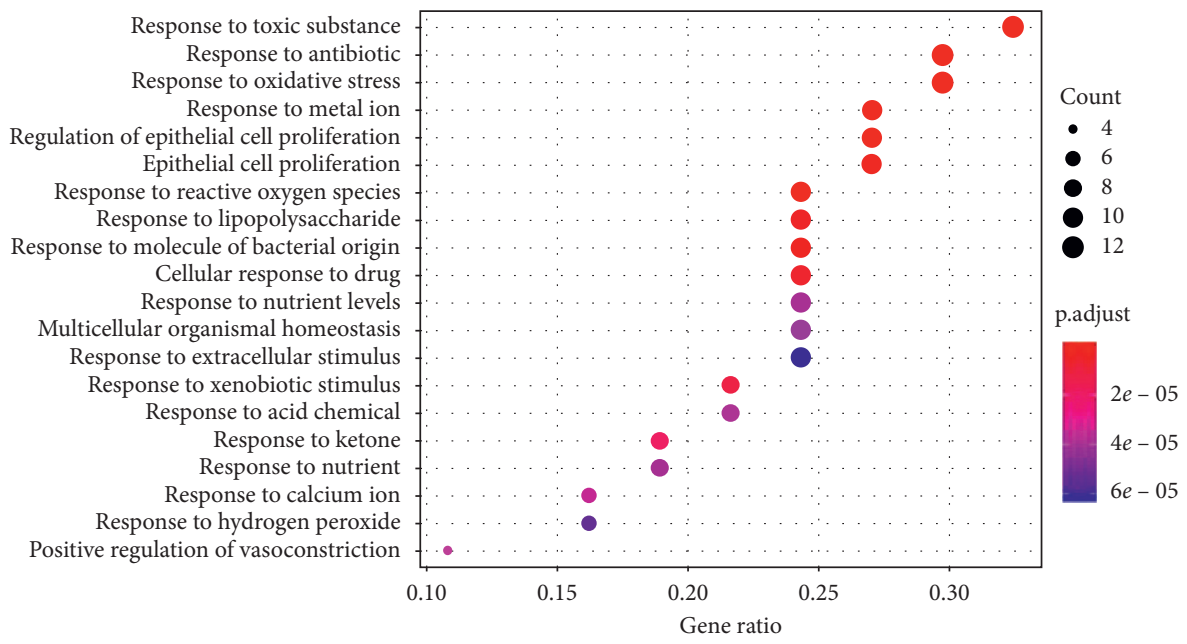

(a) 


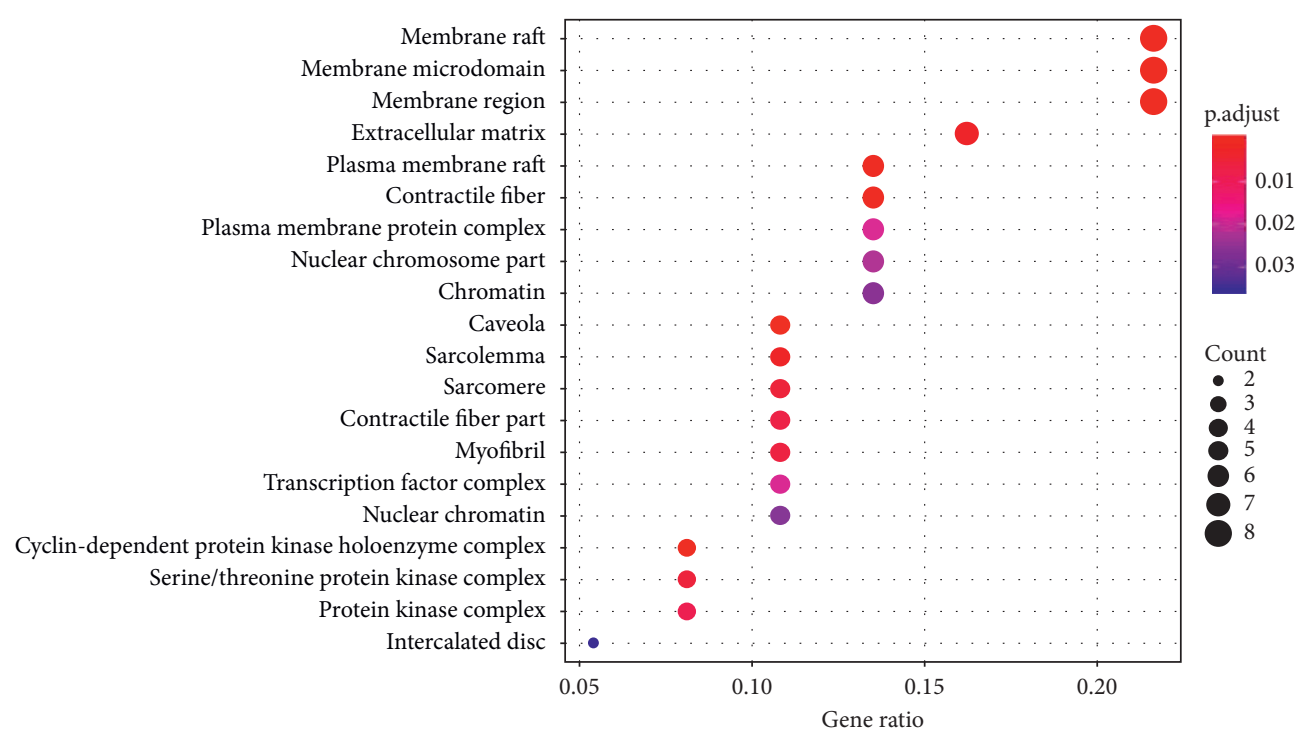

(b)

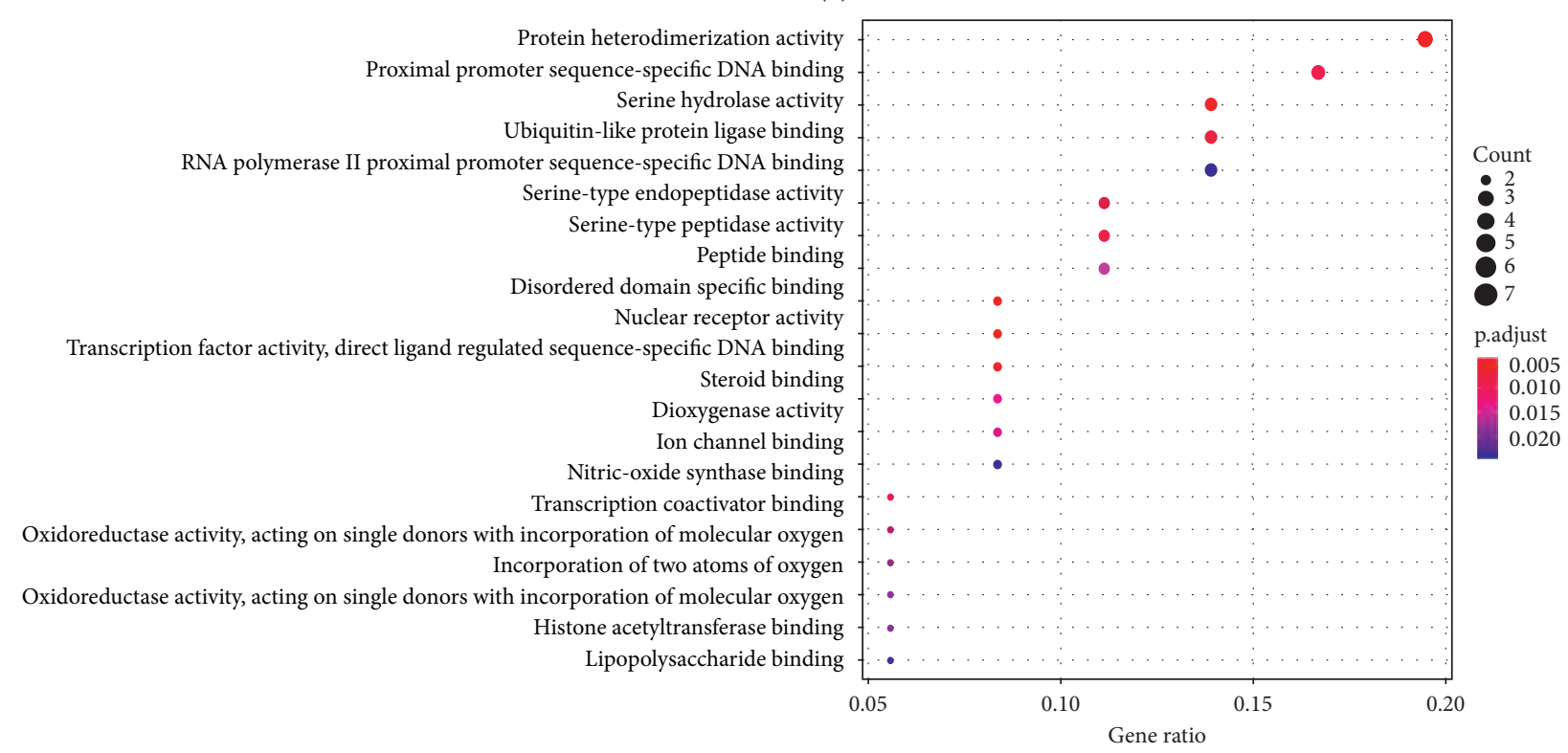

(c)

FIGURE 6: Gene ontology terms of candidate targets of the THSWT formula against ONFH. A, biological process. B, cellular component. C, molecular function.

nontraumatic ONFH. The STAT1 signaling pathway is strongly activated in the pathogenesis and progression of osteoporosis [42]. Acceleration in fracture callus remodeling and membranous ossification has been observed in STAT1-deficient mice [43].

The mechanisms of action and molecular targets of the THSWT formula for ONFH were explored using a network pharmacology approach in this study. Kaempferol, luteolin, and baicalein regulated the most number of targets associated with ONFH. The THSWT formula may regulate osteocyte function through specific BPs, including responses to toxic substances and oxidative stress. The regulated pathways include the relaxin, focal adhesion, NF- $\kappa \mathrm{B}$, TLR, and AGE/RAGE signaling pathways. RELA, VEGFA, and STAT1 


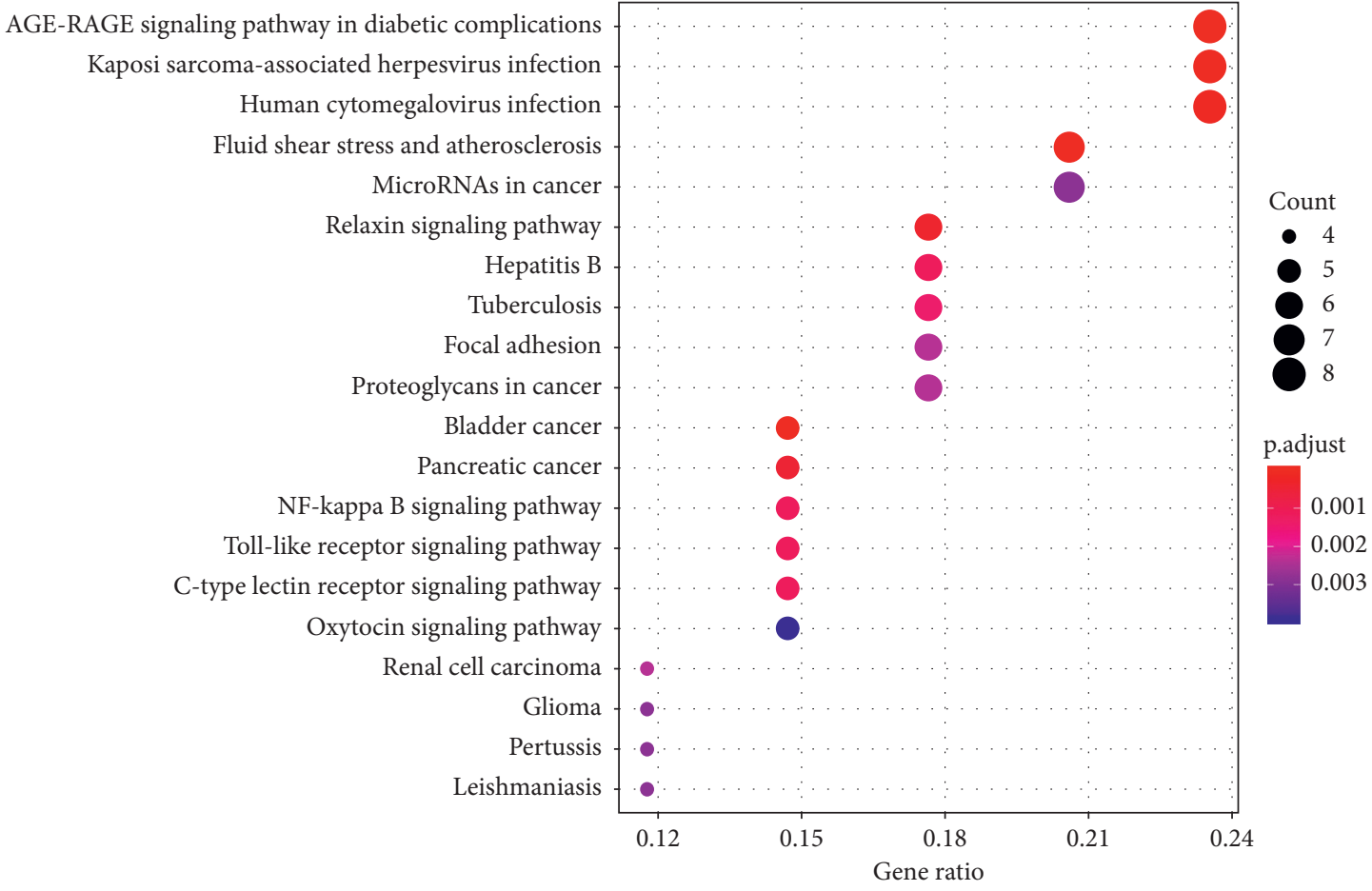

Figure 7: KEGG pathway enrichment of candidate targets of the THSWT formula against ONFH.

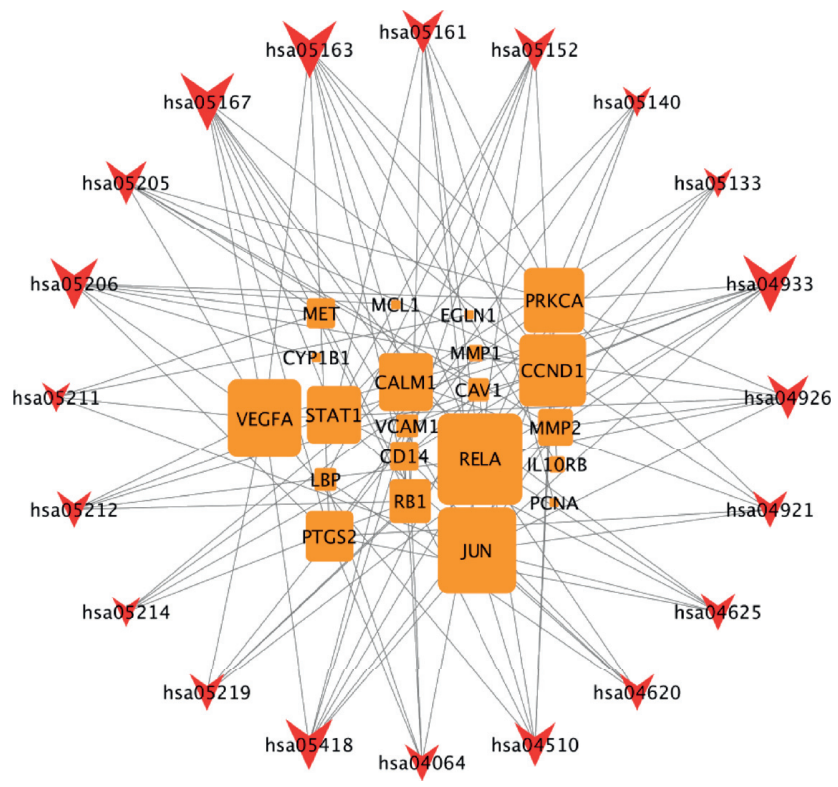

FIGURE 8: Gene-pathway network of the THSWT formula against ONFH. The orange squares represent target genes and the red V-shapes represent pathways. The importance of targets and pathways is proportional to their shape sizes.

are the important target genes in the gene network of the THSWT formula for the treatment of ONFH.

\section{Data Availability}

Raw data were generated at Wangjing Hospital. Derived data supporting the findings of this study are available from the corresponding authors on request.

\section{Disclosure}

Fanyu Fu and Zeqing Huang and should be considered cofirst authors.

\section{Conflicts of Interest}

The authors declare that they have no conflicts of interest. 


\section{Authors' Contributions}

Fanyu Fu and Zeqing Huang equally contributed to this work.

\section{Acknowledgments}

This study was supported by grants from National Natural Science Foundation of China (No. 81973888) and the Research and Development Project of the G20 Program, Beijing Municipal Commission of Science and Technology (No. Z151100003815028).

\section{References}

[1] T. Wang, B. Azeddine, W. Mah, E. J. Harvey, D. Rosenblatt, and C. Séguin, "Osteonecrosis of the femoral head: genetic basis," International Orthopaedics, vol. 43, no. 3, pp. 519-530, 2019.

[2] L. Cui, Q. Zhuang, J. Lin et al., "Multicentric epidemiologic study on six thousand three hundred and ninety five cases of femoral head osteonecrosis in China," International Orthopaedics, vol. 40, no. 2, pp. 267-276, 2016.

[3] D.-W. Zhao, M. Yu, K. Hu et al., "Prevalence of nontraumatic osteonecrosis of the femoral head and its associated risk factors in the Chinese population," Chinese Medical Journal, vol. 128 , no. 21 , pp. $2843-2850,2015$.

[4] J. Gardeniers, "Report of the committee of staging and nomenclature," ARCO News Letter, vol. 5, no. 2, pp. 79-82, 1993.

[5] M. A. Mont, M. G. Zywiel, D. R. Marker, M. S. McGrath, and R. E. Delanois, "The natural history of untreated asymptomatic osteonecrosis of the femoral head," The Journal of Bone and Joint Surgery-American Volume, vol. 92, no. 12, pp. 2165-2170, 2010.

[6] S. Kobayashi, T. Kubo, Y. Iwamoto, W. Fukushima, and N. Sugano, "Nationwide multicenter follow-up cohort study of hip arthroplasties performed for osteonecrosis of the femoral head," International Orthopaedics, vol. 42, no. 7, pp. 1661-1668, 2018.

[7] C. G. Zalavras and J. R. Lieberman, "Osteonecrosis of the femoral head," Journal of the American Academy of Orthopaedic Surgeons, vol. 22, no. 7, pp. 455-464, 2014.

[8] A. Cohen-Rosenblum and Q. Cui, "Osteonecrosis of the femoral head," Orthopedic Clinics of North America, vol. 50, no. 2, pp. 139-149, 2019.

[9] J. Wang, J. Wang, K. Zhang et al., "Bayesian network metaanalysis of the effectiveness of various interventions for nontraumatic osteonecrosis of the femoral head," BioMed Research International, vol. 2018, Article ID 2790163, 11 pages, 2018.

[10] X. Yu, D. Zhang, X. Chen, J. Yang, L. Shi, and Q. Pang, "Effectiveness of various hip preservation treatments for nontraumatic osteonecrosis of the femoral head: a network metaanalysis of randomized controlled trials," Journal of Orthopaedic Science, vol. 23, no. 2, pp. 356-364, 2018.

[11] Y. J. Lee, Q. Cui, and K.-H. Koo, "Is there a role of pharmacological treatments in the prevention or treatment of osteonecrosis of the femoral head?: A systematic review," Journal of Bone Metabolism, vol. 26, no. 1, pp. 13-18, 2019.

[12] Joint Surgery Group of the Orthopaedic Branch of the Chinese Medical Association, "Guideline for diagnostic and treatment of osteonecrosis of the femoral head," Orthopaedic Surgery, vol. 7, no. 3, pp. 200-207, 2015.
[13] D. Zhao, F. Zhang, B. Wang et al., "Guidelines for clinical diagnosis and treatment of osteonecrosis of the femoral head in adults (2019 version)," Journal of Orthopaedic Translation, vol. 21, pp. 100-110, 2020.

[14] D.-w. Zhao and Y.-c. Hu, “Chinese experts' consensus on the diagnosis and treatment of osteonecrosis of the femoral head in adults," Orthopaedic Surgery, vol. 4, no. 3, pp. 125-130, 2012.

[15] Microsurgery Department of the Orthopedics Branch of the Chinese Medical Doctor Association, "Chinese guideline for the diagnosis and treatment of osteonecrosis of the femoral head in adults," Orthopaedic Surgery, vol. 9, no. 1, pp. 3-12, 2017.

[16] X. Guo, W. Chen, and Y. Zhao, "The three-stage syndrome differentiation systerm for aseptic osteonecrosis of the femoral head: a case series study of 50 patients," in Proceedings of the China Symposium on Aseptic Osteonecrosis of the Femoral Head, pp. 310-313, Beijing, China, 1992.

[17] J. Wu, L. Yao, B. Wang, Z. Liu, and K. Ma, "Tao-Hong-Si-Wu Decoction ameliorates steroid-induced avascular necrosis of the femoral head by regulating the HIF- $1 \alpha$ pathway and cell apoptosis," BioScience Trends, vol. 10, no. 5, pp. 410-417, 2016.

[18] J. Ru, P. Li, J. Wang et al., "TCMSP: a database of systems pharmacology for drug discovery from herbal medicines," Journal of Cheminformatics, vol. 6, p. 13, 2014.

[19] D. S. Wishart, C. Knox, A. C. Guo et al., "DrugBank: a comprehensive resource for in silico drug discovery and exploration," Nucleic Acids Research, vol. 34, no. 90001, pp. D668-D672, 2006.

[20] M. Soudy, A. M. Anwar, E. A. Ahmed et al., "UniprotR: retrieving and visualizing protein sequence and functional information from universal protein resource (uniprot knowledge base)," Journal of Proteomics, vol. 213, Article ID 103613, 2020.

[21] E. Clough and T. Barrett, "The gene expression omnibus database," Methods in Molecular Biology, vol. 1418, pp. 93110, 2016.

[22] W. Larry, "Practical extraction and report language (perl)," 2008.

[23] C. Zhao, J. Chen, B. Yu et al., "Effect of modified taohongsiwu decoction on patients with chemotherapy-induced hand-foot syndrome," Journal of Traditional Chinese Medicine = Chung I Tsa Chih Ying Wen pan, vol. 34, no. 1, pp. 10-14, 2014.

[24] W. Bao, Y. Sun, and L. Yan, "Clinical observation on the treatment of early osteonecrosis of the femoral head with extracorporeal shock wave combining modified taohong siwu tang," Rheumatism and Arthritis, vol. 5, no. 5, pp. 24-26, 2016.

[25] Z.-R. Li, L.-M. Cheng, K.-Z. Wang et al., "Herbal Fufang Xian Ling Gu Bao prevents corticosteroid-induced osteonecrosis of the femoral head-A first multicentre, randomised, doubleblind, placebo-controlled clinical trial," Journal of Orthopaedic Translation, vol. 12, pp. 36-44, 2018.

[26] Q.-S. Wei, G.-J. Hong, Y.-J. Yuan, Z.-Q. Chen, Q.-W. Zhang, and W. He, "Huo Xue Tong Luo capsule, a vasoactive herbal formula prevents progression of asymptomatic osteonecrosis of femoral head: a prospective study," Journal of Orthopaedic Translation, vol. 18, pp. 65-73, 2019.

[27] M. Imran, A. Rauf, Z. A. Shah et al., "Chemo-preventive and therapeutic effect of the dietary flavonoid kaempferol: a comprehensive review," Phytotherapy Research, vol. 33, no. 2, pp. 263-275, 2019.

[28] S. Adhikary, D. Choudhary, N. Ahmad et al., "Dietary flavonoid kaempferol inhibits glucocorticoid-induced bone loss 
by promoting osteoblast survival," Nutrition, vol. 53, pp. 64-76, 2018.

[29] N. Aziz, M.-Y. Kim, and J. Y. Cho, "Anti-inflammatory effects of luteolin: a review of in vitro, in vivo, and in silico studies," Journal of Ethnopharmacology, vol. 225, pp. 342-358, 2018.

[30] J.-W. Lee, J.-Y. Ahn, S.-i. Hasegawa et al., "Inhibitory effect of luteolin on osteoclast differentiation and function," Cytotechnology, vol. 61, no. 3, pp. 125-134, 2009.

[31] K. Sowndhararajan, P. Deepa, M. Kim, S. J. Park, and S. Kim, "Baicalein as a potent neuroprotective agent: a review," Biomedicine \& Pharmacotherapy, vol. 95, pp. 1021-1032, 2017.

[32] M. H. Kim, S. Y. Ryu, M. A. Bae, J.-S. Choi, Y. K. Min, and S. H. Kim, "Baicalein inhibits osteoclast differentiation and induces mature osteoclast apoptosis," Food and Chemical Toxicology, vol. 46, no. 11, pp. 3375-3382, 2008.

[33] Y. Zhang, X. Kong, R. Wang et al., "Genetic association of the P-glycoprotein gene ABCB1 polymorphisms with the risk for steroid-induced osteonecrosis of the femoral head in Chinese population," Molecular Biology Reports, vol. 41, no. 5, pp. 3135-3146, 2014.

[34] K. Tyml, "Critical role for oxidative stress, platelets, and coagulation in capillary blood flow impairment in sepsis," Microcirculation, vol. 18, no. 2, pp. 152-162, 2011.

[35] A. Ferlin, A. Pepe, A. Facciolli, L. Gianesello, and C. Foresta, "Relaxin stimulates osteoclast differentiation and activation," Bone, vol. 46, no. 2, pp. 504-513, 2010.

[36] M. A. Shelef, D. A. Bennin, N. Yasmin et al., "Focal adhesion kinase is required for synovial fibroblast invasion, but not murine inflammatory arthritis," Arthritis Research \& Therapy, vol. 16, no. 5, p. 464, 2014.

[37] L. Tian, Q. Wen, X. Dang et al., "Immune response associated with Toll-like receptor 4 signaling pathway leads to steroidinduced femoral head osteonecrosis," BMC Musculoskeletal Disorders, vol. 15, p. 18, 2014.

[38] S.-i. Yamagishi, K. Nakamura, T. Matsui, S. Ueda, K. Fukami, and S. Okuda, "Agents that block advanced glycation end product (AGE)-RAGE (receptor for AGEs)-oxidative stress system: a novel therapeutic strategy for diabetic vascular complications," Expert Opinion on Investigational Drugs, vol. 17, no. 7, pp. 983-996, 2008.

[39] L. I. Plotkin, A. L. Essex, and H. M. Davis, "RAGE signaling in skeletal biology," Current Osteoporosis Reports, vol. 17, no. 1, pp. 16-25, 2019.

[40] S. Vaira, M. Alhawagri, I. Anwisye et al., "RelA/p65 promotes osteoclast differentiation by blocking a RANKL-induced apoptotic JNK pathway in mice," The Journal of Clinical Investigation, vol. 118, no. 6, pp. 2088-2097, 2008.

[41] W. Ma, K. Xin, K. Chen et al., "Relationship of common variants in VEGFA gene with osteonecrosis of the femoral head: a Han Chinese population based association study," Scientific Reports, vol. 8, no. 1, p. 16221, 2018.

[42] X. Ma, Z. Xu, S. Ding et al., "Alendronate promotes osteoblast differentiation and bone formation in ovariectomy-induced osteoporosis through interferon- $\beta /$ signal transducer and activator of transcription 1 pathway," Experimental and Therapeutic Medicine, vol. 15, no. 1, pp. 182-190, 2018.

[43] K. Tajima, H. Takaishi, J. Takito et al., "Inhibition of STAT1 accelerates bone fracture healing," Journal of Orthopaedic Research, vol. 28, no. 7, pp. 937-941, 2010. 